\title{
Diffusion-weighted imaging: As the first diagnostic clue to Creutzfeldt Jacob disease
}

\author{
Sunitha P Kumaran, Kanchan Gupta, Pushpa BT, Sanjaya Viswamitra, EV Joshy ${ }^{1}$ \\ Departments of Radiology and ${ }^{1}$ Neurology, Sri Sathya Sai Institute of Higher Medical Sciences, Bangalore, Karnataka, India
}

\section{ABSTRACT}

We describe a case report where the diagnosis of sporadic Creutzfeldt-Jakob disease(CJD) was suspected by the magnetic resonance imaging (MRI) radiologist in a 75-year-old lady who presented with rapid cognitive decline. MRI revealed cortical T2 and FLAIR(fluid attenuation and inversion recovery) hyperintensities in bilateral fronto-parietal and temporo-occipital locations and showed significant restriction on diffusion-weighted images(DWI). In this case report, we discuss the role of MRI to suspect the diagnosis of CJD in appropriate clinical settings.

Key words: Creutzfeldt-Jacob disease, diffusion weighted imaging

\section{Introduction}

Creutzfeldt-Jakob disease(CJD) is a subacutely progressive neurological disorder caused by deposition of prion proteins in brain. It is a very rare entity and the world-wide incidence is $0.5-1.0$ cases per million per year. ${ }^{[1]}$ A majority of cases are sporadic, some cases are familial, carrying a prion protein mutation and it is usually fatal within 1 year. ${ }^{[2]}$ We report the significance of MRI findings to correlate and support the clinical diagnosis of CJD.

\section{Case Report}

A 75 year-old lady presented with rapid cognitive decline of three months duration. A patient was evaluated as a case of dementia and had frontal, parietal, temporal, and occipital deficit signs on neuro-psychiatry evaluation. MRI was done as a part of her investigation on a 1.5T GE (Signa, USA) scanner, which showed diffuse cortical T2 and FLAIR hyperintensities in bilateral frontal, parietal,

\begin{tabular}{|l|l|}
\hline \multicolumn{2}{|c|}{ Access this article online } \\
\hline Quick Response Code: & Website: \\
\cline { 1 - 2 } & www.ruralneuropractice.com \\
\cline { 2 - 3 } & \\
\hline
\end{tabular}

temporal, and occipital locations [Figures 1 and 2]. The cortical hyperintensities showed significant restriction on

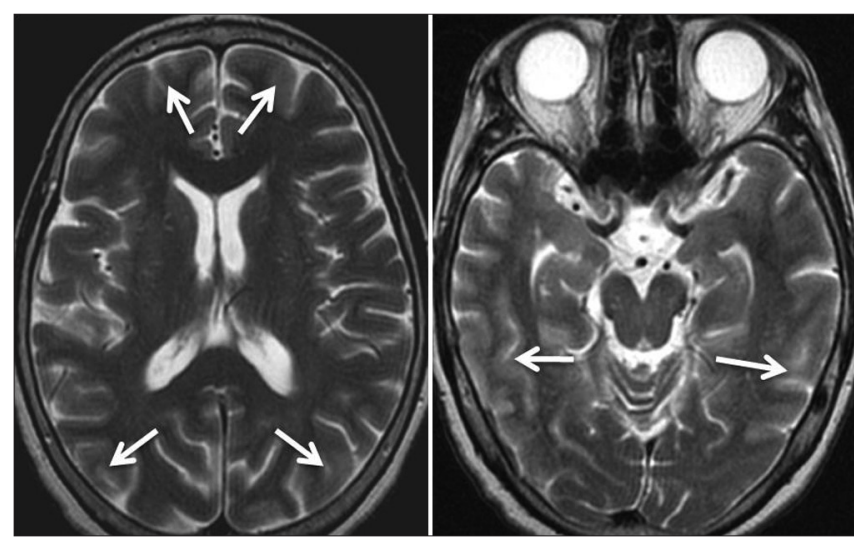

Figure 1: Axial T2 W MR images showing faint and equivocal cortical hyperintensities (arrows) in bilateral frontal, parietal, and temporal lobes.
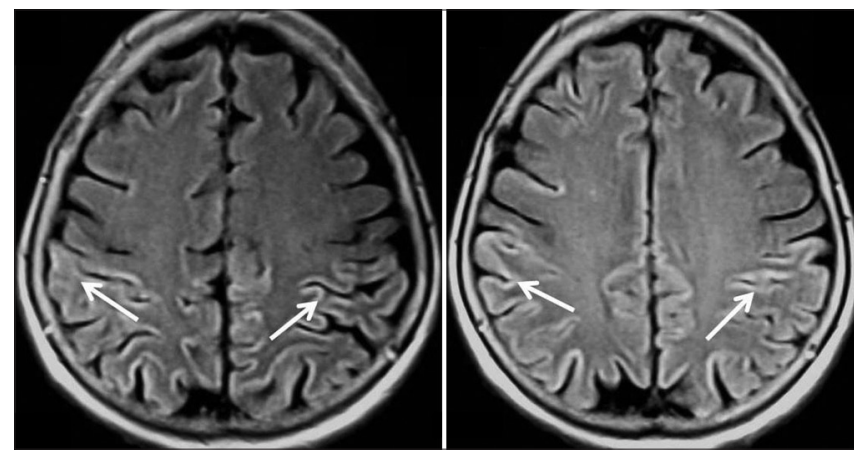

Figure 2: Axial FLAIR MR images shows the signal abnormalities slightly better compared to Figure 1, although cortical hyperintensities (arrows) are not very striking.

\section{Address for correspondence:}

Dr. Sunitha P Kumaran, Department of Radiology, Sri Sathya Sai Institute of Higher Medical Sciences, EPIP area, Whitefield, Bangalore-560066, Karnataka, India. E-mail: drsunitha27@gmail.com 
DWI [Figure 3], giving rise to the strikingly conspicuous lesions compared to the normal cortex signal in the above locations.

The radiologist first suspected the diagnosis and alerted the clinician. Later in the course of the disease, she developed myoclonic jerks, EEG changes which were consistent with the WHO criteria of probable CJD. The patient died in 6 months from the onset of symptoms.

\section{Discussion}

According to the national CJD registry at NIMHANS, Bangalore, India, there are only 85 recorded cases of CJD till September 2005. ${ }^{[3]}$ The diagnostic triad of CJD i.e., of a progressive dementia, myoclonus and periodic sharp wave EEG activity may not be evident in about $25 \%$ of the cases. ${ }^{[4]}$

MRI with DW and FLAIR sequences is an invaluable modality in supporting the diagnosis of CJD. ${ }^{[5]}$ Shiga et $a^{[6]}$ in his study concluded that diffusion-weighted $\operatorname{MRI}(92 \%)$ had higher sensitivity in the detection of CJD than FLAIR sequences (41-59\%), T2 (36-50\%), EEG (50-78\%), CSF protein $14-3-3(84 \%)$ or neuron-specific enolase (73\%). Diffusion-weighted MRI is more resistant for motion artifacts than T2W and FLAIR images. ${ }^{[6]}$ The abnormalities on DW-MRI could be detected as early as at 3 weeks of symptom duration and even before the appearance of periodic triphasic waves on EEG. ${ }^{[6]}$ Matoba et $a l^{[7]}$ noted that the hyperintensity in the basal ganglia and cortex during the early stages was more extensive and conspicuous while in the later stages there was disappearance of the abnormal signals in the cortex.

The cause for restricted diffusion is attributed to accumulation of abnormal vacuoles in the cytoplasm and microvacuolation of neuritic process heralding spongiform degeneration. ${ }^{[8]}$ MRI also helps in differentiating sporadic CJD from variant CJD. ${ }^{8]}$ Sporadic CJD shows T2 prolongation and restricted diffusion in the corpus striatum followed by the neocortex and posterior and medial thalami. ${ }^{[2]}$ In variant-CJD, the T2 hyperintensities are commonly located in the posterior (pulvinar) and medial thalami followed by periaqueductal grey matter, striatum, and less commonly in the neo-cortex. ${ }^{[6]}$ The increased signal intensity in the pulvinar relative to the anterior putamen is called the "Pulvinar sign" is the most sensitive marker for variant CJD. ${ }^{[5]}$ The bilateral pulvinar sign has a sensitivity of $78 \%$ and correlates with the histological gliosis. ${ }^{[5]}$

The combination of FLAIR and DW imaging has a sensitivity, specificity and accuracy of over $90 \%$ in differentiating CJD from other dementias. ${ }^{[9]}$ The multifocal cortical and subcortical hyperintensities in the grey matter showing restricted diffusion on MRI may be more useful than the CSF protein 14-3-3 analysis. ${ }^{[10]}$ 14-3-3 protein analysis was not performed because of the lack of the availability of the test at our institute. CSF protein 14-3-3 analysis availability is limited only to a few cities and is not done routinely in all the hospitals. However, diagnosis can only be confirmed by histological examination of brain tissue obtained either by the brain biopsy or after autopsy. These are not performed by many institutions due to the transmissible nature of the disease.

\section{Conclusion}

This case highlights the role of DW-MRI as practical modality in the early diagnosis of CJD. MRI Radiologist can give the first clue to an unsuspected case of CJD,

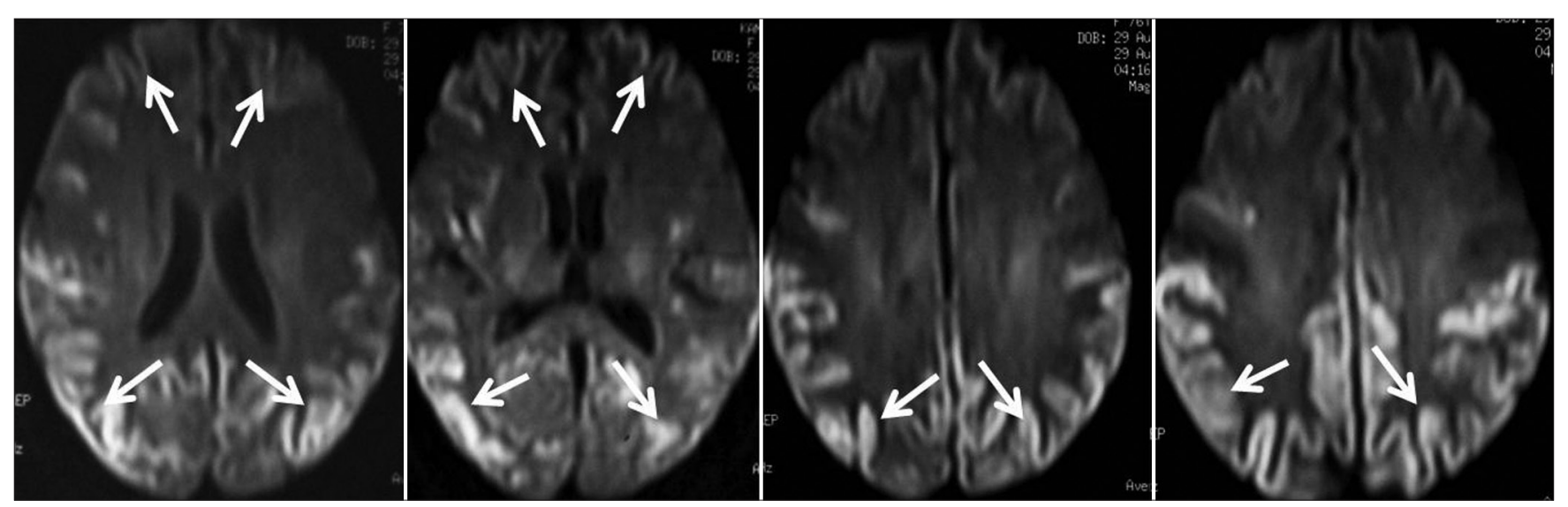

Figure 3: Diffusion weighted MR images clearly shows increased visibility of high signal from restricted diffusion (arrows) in the bilateral frontal, parietal, temporal, and occipital regions along the cortical locations. 
as in this case and can make it easy for the Clinician who has to do an elaborate work-up of dementia cases. Radiologists and neurologists should be familiar with the characteristic appearance of DW imaging as a clue to the diagnosis of this dreaded disease, for which no treatment is available till date. In a patient with rapid dementia, MRI should be done as it is accurate, noninvasive, and cost-effective (as compared to CSF-14-3-3 analysis) and if findings are suggestive of CJD, the clinician should be alarmed and appropriate infection control measures are to be taken.

\section{References}

1. Mehndiratta MM, Bajaj BK, Gupta M, Anand R, Tatke M, Seryam S, et al. Creutzfeldt-Jakob disease: Report of 10 cases from North India. Neurol India 2002; 50:113.

2. Praveen KS, Sinha S, Chandrasekhar HS, Vijayan J, Taly AB. Sporadic onset Creutzfeldt-Jacob disease: Interesting MRI observations. Neurol India 2006;54:418-20.

3. Shankar SK, Satishchandra P. Did BSE in the UK originate from Indian continent? Lancet 2005;366:790-1.

4. Johnson RT, Gibbs CJ Jr. Creutzfeldt-Jakob disease and related transmissible spongiform encephalopathies. N Engl J Med 1998;339: 1994-2004.

5. Zeidler M, Sellar RJ, Collie DA, Knight R, Stewart G, Macleod MA, et al. The pulvinar sign on magnetic resonance imaging in variant CreutzfeldtJakob disease. Lancet 2000;355:1412-8.

6. Kandiah N, Tan K, Pan AB, Au WL, Venketasubramanian N, and Tchoyoson Lim CC, et al. Creutzfeldt - Jakob disease: Which diffusionweighted imaging abnormality is associated with periodic EEG complexes? Neurol 2008;255:1411-4.

7. Matoba M, Tonami H, Miyagi H, Yokota H, Yamamoto I. CreutzfeldtJakob Disease: Serial changes on diffusion weighted MRI. J Comput Assist Tomogr 2001;25:274-7.

8. Schroter A, Zerr I, Henkel K, Tschampa HJ, Finkenstaedt M, Poser S. Magnetic resonance imaging in the clinical diagnosis of Creutzfeldt-Jakob disease. Arch Neurol 2000;57:1751-7.

9. Young GS, Geschwind DM, Fischbein JN, Martindale LJ, Henry GR, Liu S, et al. Diffusion weighted and fluid attenuated inversion recovery imaging in Creutzfeldt-Jakob disease: High sensitivity and specificity for diagnosis. Am J Neuroradiol 2005;26:1551-62.

10. Mendez OE, Shang J, Jungreis CA, Kaufer DI. Diffusion weighted MRI in CJD is a better diagnostic marker than CSF protein 14-3-3. J Neuroimaging 2003;13:147-51.

How to cite this article: Kumaran SP, Gupta K, Pushpa BT, Viswamitra S, Joshy EV. Diffusion-weighted imaging: As the first diagnostic clue to Creutzfeldt Jacob disease. J Neurosci Rural Pract 2012;3:408-10.

Source of Support: Nil. Conflict of Interest: None declared. 